\title{
Phytoprotection
}

\section{Première mention du Marssonina salicicola sur des saules pleureurs au Québec}

\section{First mention of Marssonina salicicola on weeping willows in the province of Québec}

\author{
V. Vujanovic, M. St-Arnaud et P. Neumann
}

Volume 79, numéro 2, 1998

URI : https://id.erudit.org/iderudit/706137ar

DOI : https://doi.org/10.7202/706137ar

Aller au sommaire du numéro

Éditeur(s)

Société de protection des plantes du Québec (SPPQ)l

ISSN

0031-9511 (imprimé)

1710-1603 (numérique)

Découvrir la revue

Citer cet article

Vujanovic, V., St-Arnaud, M. \& Neumann, P. (1998). Première mention du Marssonina salicicola sur des saules pleureurs au Québec. Phytoprotection, 79(2), 87-91. https://doi.org/10.7202/706137ar
Résumé de l'article

Au cours des printemps 1996 et 1997, des prélèvements ont été réalisés sur cinq sites à Montréal (Québec) pour établir l'étiologie de l'anthracnose des saules pleureurs (Salix alba Tristis' et S. babylonica). Sur les 25 arbres examinés, 21 étaient affectés par une maladie fongique due au Marssonina salicicola qui constitue le stade conidien du Drepanopeziza sphaeroides. Cette détection est la première mention de la présence du champignon au Canada, à l'est des Rocheuses. 


\title{
Communication brève / Short Communication Première mention du Marssonina salicicola sur des saules pleureurs au Québec
}

\author{
Vladimir Vujanovic ${ }^{1,2}$, Marc St-Arnaud ${ }^{1}$ et Peterjürgen \\ Neumann ${ }^{2}$
}

Reçu 1998-05-25; accepté 1998-11-20

PHYTOPROTECTION $79: 87-91$

Au cours des printemps 1996 et 1997, des prélèvements ont été réalisés sur cinq sites à Montréal (Québec) pour établir l'étiologie de l'anthracnose des saules pleureurs (Salix alba 'Tristis' et $S$. babylonica). Sur les 25 arbres examinés, 21 étaient affectés par une maladie fongique due au Marssonina salicicola qui constitue le stade conidien du Drepanopeziza sphaeroides. Cette détection est la première mention de la présence du champignon au Canada, à l'est des Rocheuses.

\section{[First mention of Marssonina salicicola on weeping willows in the province of Quebec]}

During spring 1996 and 1997, five sites on the Island of Montreal, Quebec, were examined to study the occurrence of anthracnose on weeping willows (Salix alba 'Tristis' and S. babylonica). On 21 trees of 25 surveyed, the pathogenic fungus Marssonina salicicola, the conidial state of Drepanopeziza sphaeroides, was found. This report is the first mention of the presence of this fungus in Canada, east of the Rocky Mountains.

Un des agents pathogènes le plus dévastateur des saules pleureurs est le coelomycète Marssonina salicicola (Bres.) Magnus (Meirleire 1978), forme conidienne du Drepanopeziza sphaeroides (Pers.) Höhnel (Helotiales), démontré par Rimpau (1961). II a été signalé sur plusieurs espèces et formes de saules, dont les Salix alba L. 'Tristis', $S$. alba 'Vitellina', S. americana Hort., $S$. angustifolia B. et G., S. babylona L., S. caprea L., S. coerulea Rub., S. daphnoides Vill., S. elegantissima Koch, S. fragilis L. et S. purpurea L. (Butin 1983; Vegh 1974). Depuis la première description en Allemagne, en 1893, par Bresadola, ce parasite a été identifié sur tous les continents, particulièrement dans les régions au climat humide et tempéré (Farr et al. 1989; Marks et al. 1982; Nattrass 1930; Siskina et Mamykasvili 1978; Tzavella-Klonari et al. 1997; Vegh et Bourgeois 1968; Vujanovic et Vucinic 1992). Au Canada, Hill et al. (1977) ont constaté que plus de $70 \%$ des saules pleureurs, dans la zone côtière du sud de la Colombie-Britannique, étaient infectés par le $M$. salicicola. À l'est des Rocheuses canadiennes, le M. salicicola n'a, jusqu'à ce jour, jamais été signalé. Par contre, le M. kriegeriana (Bres.) Magnus, fréquemment confondu avec le $M$. salicicola (Vegh et Velastegui 1983), est mentionné comme agent causal de l'anthracnose chez le $S$. alba 'Tristis' et le S. babylonica (Conners

1. Institut de recherche en biologie végétale, Université de Montréal et Jardin botanique de Montréal, 4101, rue Sherbrooke Est, Montréal (Québec), Canada H1X 2B2

2. Département de sciences biologiques, Université de Montréal, C.P. 6128, Succursale Centre-ville, Montréal (Québec), Canada H3C 3J7 
1967). Puisque la maladie est répandue dans les jardins et les plantations à Montréal (Québec), I'objectif de cette étude était de clarifier l'identité de l'organisme responsable et de mettre en évidence son étiologie.

Nous avons examiné en 1996 et en 1997, sur l'Île de Montréal (Québec), 25 saules pleureurs. Sur les 21 arbres montrant des signes d'anthacnose, nous avons effectué 8 à 10 prélèvements en échantillonnant à chaque prélèvement 20 feuilles et 10 portions de rameaux de $20 \mathrm{~cm}$ de longueur par arbre. La fréquence de l'échantillonnage a été fixée à des intervalles de $14 \mathrm{j}$ durant le printemps et de deux mois pendant le reste de l'année. D'après la technique de Vegh (1974), nous avons récolté des conidies à partir des gelées sporifères sur des jeunes lésions pour préparer des suspensions de conidies diluées à 10000 spores $\mathrm{mL}^{-1}$. Une goutte de suspension de $20 \mu \mathrm{L}$ était placée sur de l'eau gélosée $(1,5 \%)$ au centre d'une boîte de Petri. Après $48 \mathrm{~h}$ d'incubation à $20^{\circ} \mathrm{C}$, deux disques de gélose $\left(20 \mathrm{~mm}^{2}\right)$ par boîte de Petri, portant des spores en germination, ont été repiqués sur une gélose nutritive (PDA $2 \%$ ) pour initier la croissance mycélienne. Parallèlement, la présence de ramifications des filaments germinatifs a été observée en boîte de Petri à l'aide d'un microscope Carl Zeiss $(40 \mathrm{x})$. L'influence de la température (valeurs cardinales) sur la croissance mycélienne a été évaluée sur PDA (2 \%).

Les observations sur les saules pleureurs malades indiquent qu'il s'agit du $M$. salicicola et non du M. kriegeriana, responsable de I'anthracnose (Fig. 1 ae): 1) les feuilles parasitées se transforment, elles deviennent rabougries et recroquevillées. Pour l'ensemble, elles restent plus petites que les feuilles non parasitées. Par contre, $M$. kriegeriana ne provoque pas une déformation foliaire (Vegh et Velastegui 1983); 2) les lésions observées - d'une taille de 3 à $6 \mathrm{~mm}$ - sont présentes aussi bien sur les feuilles que sur les rameaux. Par contre, $M$. kriegeriana cause des taches plus petites et exclusivement sur les feuilles (Tableau 1) (Vegh et Velastegui 1983).
Nos observations concordent très bien avec des descriptions antérieures (Lanier et al. 1976; Nival 1972; Vegh et Velastegui 1983), aussi bien pour la symptomatologie que pour la morphologie et la formation des acervules, qui sont produits au début du printemps à partir du parenchyme cortical des rameaux ou au moment de la formation des taches sur les feuilles.

Nos résultats des observations microscopiques et culturales sont également conformes à la description de $M$. salicicola (Fig. $1 \mathrm{f}-\mathrm{h}$ ) (Vegh et Velastegui 1983). Les macroconidies sont piriformes ou subovoïdes (13-18 x 5-8 $\mu \mathrm{m})$ et les microconidies en forme de bâtonnets $(2-4 \times 1,2 \mu \mathrm{m})$. Indépendamment de la taille et de la forme des macroconidies d'été, il y a des caractères qui distinguent très nettement les deux champignons: 1) la formation des "conidies secondaires" à partir des macroconidies observées chez nos isolats a lieu seulement chez $M$. salicicola; 2) le taux de germination est faible en présence d'eau chez $M$. salicicola et 3 ) les hyphes germinatifs de $M$. salicicola se ramifient rapidement, contrairement à ceux de $M$. kriegeriana. Tous ces caractères sont présents chez les champignons isolés qui correspondent ainsi à $M$. salicicola et non à $M$. kriegeriana (Tableau 1), et l'isolation de ce champignon a été possible à partir de tous les 21 arbres montrant les symptômes d'anthracnose. Occasionnellement, à partir des feuilles mortes, un Mycosphaerella sp. a aussi pu être détecté. De plus, sur des rameaux affectés par le $M$. salicicola et qui meurent quelques mois plus tard, on observe le développement sporadique de fructifications de Cytospora sp. et de Diplodina sp.

II faut donc conclure que l'anthracnose des saules pleureurs sur l'île de Montréal est principalement provoquée par le $M$. salicicola et que son identification dans cette région représente une première mention pour l'Est du Canada. Depuis, nous avons également trouvé ce champignon à Berthierville (Québec), $80 \mathrm{~km}$ à l'est de Montréal. II reste enfin à prouver que le $M$. kriegeriana est véritablement présent dans la région. 


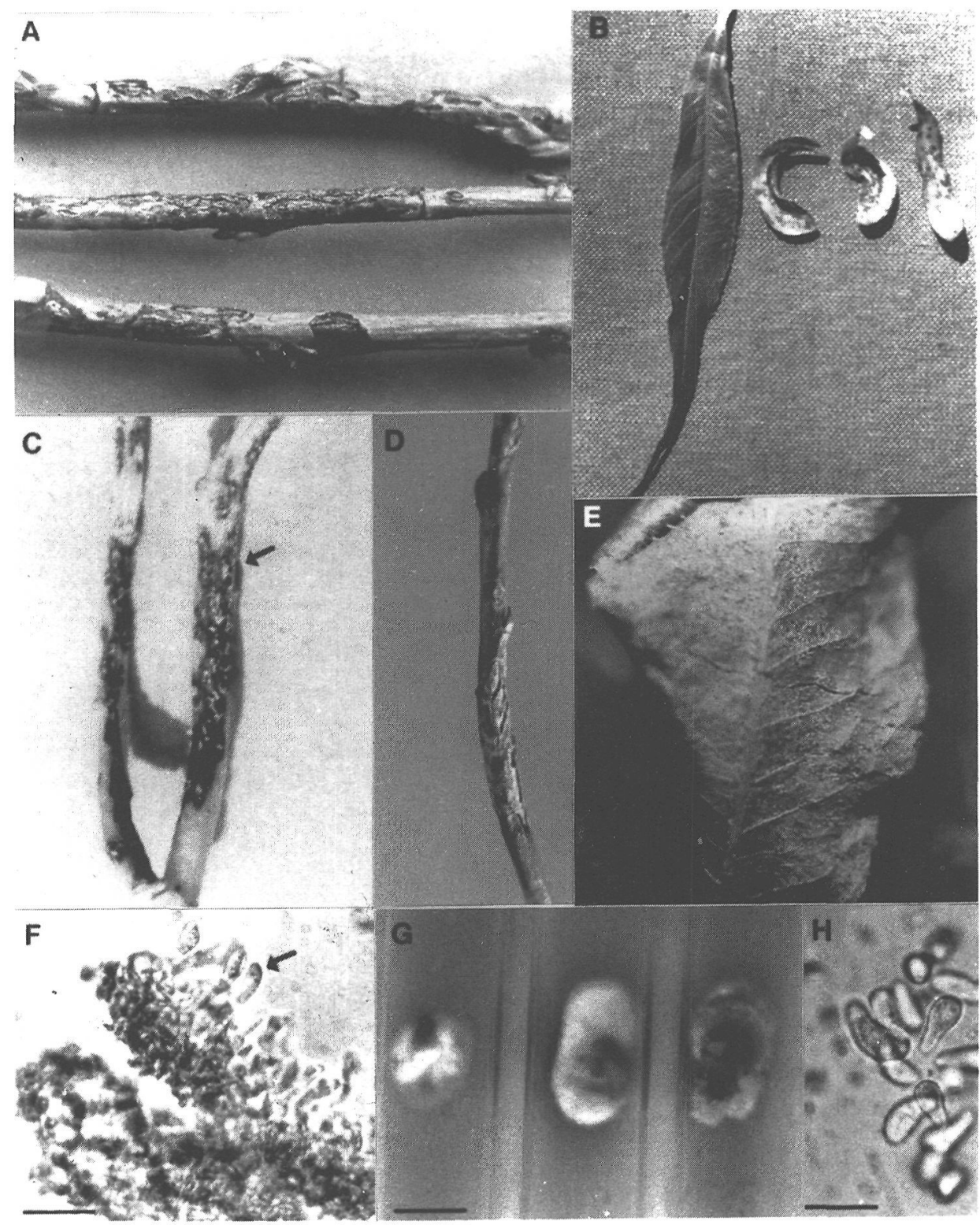

Figure 1. L'anthracnose des saules pleureurs causée par $M$. salicicola. A) dégâts caractéristiques sur les rameaux d'une année; $B$ ) déformation des jeunes feuilles; C) lésion sur les jeunes rameaux porteurs de gelées sporifères (durant le printemps); D) chancre et déformation du rameau (durant l'été); E) feuille morte avec acervules (durant l'hiver); F) coupe transversale d'acervule avec les macroconidies estivales; G) cultures du $M$. salicicola après 10, 20 et 30 j sur milieu PDA (échelle: $1 \mathrm{~cm}$ ); H) macroconidies du $M$. salicicola sur milieu PDA (échelle : $15 \mu \mathrm{m})$. 
Tableau 1. Caractères importants' ayant servi pour l'identification du Marssonina salicicola, basée sur les données du tableau 1 de Vegh et Velastegui (1983) comparant $M$. kriegeriana et $M$. salicicola

\begin{tabular}{|c|c|c|c|}
\hline Critères à étudier & $\begin{array}{l}\text { Marssonina } \\
\text { kriegeriana }\end{array}$ & $\begin{array}{l}\text { Marssonina } \\
\text { salicicola }\end{array}$ & $\begin{array}{l}\text { Caractères } \\
\text { observés }\end{array}$ \\
\hline $\begin{array}{l}\text { Organes végétaux } \\
\text { attaqués }\end{array}$ & Essentiellement les feuilles & $\begin{array}{l}\text { Inflorescences, feuilles, } \\
\text { rameaux }\end{array}$ & $\sqrt{ }$ \\
\hline $\begin{array}{l}\text { Symptômes sur } \\
\text { les feuilles }\end{array}$ & $\begin{array}{l}\text { Petites taches } \pm \text { arrondies } \\
\text { à contour régulier ou } \\
\text { fibrilleux; centre clair, bord } \\
\text { brun ou marron à rosâtre; } \\
\text { pas de réduction ni de } \\
\text { déformation des feuilles }\end{array}$ & $\begin{array}{l}\text { Petites taches } \pm \text { arrondies de } \\
\text { couleur brun rouge, dont le } \\
\text { centre devient grisâtre; } \\
\text { réduction et déformation des } \\
\text { feuilles }\end{array}$ & $\checkmark$ \\
\hline Manifestation des dégâts & $\begin{array}{l}\text { Dès la fin du printemps } \\
\text { jusqu'à la chute des } \\
\text { feuilles: principalement fin } \\
\text { d'été et début d'automne }\end{array}$ & $\begin{array}{l}\text { Dès l'apparition des feuilles } \\
\text { et durant toute la période de } \\
\text { végétation: principalement fin } \\
\text { de printemps et début d'été }\end{array}$ & $\checkmark$ \\
\hline $\begin{array}{l}\text { Morphologie des } \\
\text { "macroconidies d'été» }\end{array}$ & $\begin{array}{l}\text { Piriformes ou subovoïdes, } \\
\text { presque toutes les } \\
\text { macroconidies sont } \\
\text { faiblement courbées }\end{array}$ & $\begin{array}{l}\text { Piriformes ou subovoïdes, } \\
\text { plus de } 50 \% \text { des } \\
\text { macroconidies faiblement } \\
\text { courbées }\end{array}$ & $\checkmark$ \\
\hline $\begin{array}{l}\text { Germination des } \\
\text { macroconidies sur l'eau } \\
\text { gélosée par "conidies } \\
\text { secondaires" }\end{array}$ & Absentes & Fréquentes & $\sqrt{ }$ \\
\hline $\begin{array}{l}\text { Germination des } \\
\text { macroconidies en } \\
\text { fonction de la } \\
\text { température sur l'eau } \\
\text { gélosée }\end{array}$ & Optimum: $20^{\circ} \mathrm{C}$ & Optimum: $22^{\circ} \mathrm{C}$ & $\checkmark$ \\
\hline $\begin{array}{l}\text { Germination des } \\
\text { macroconidies en } \\
\text { présence d'eau liquide }\end{array}$ & Assez importante (50-60\%) & Très faible $(1-10 \%)$ & $\sqrt{ }$ \\
\hline $\begin{array}{l}\text { Ramification des } \\
\text { filaments germinatifs sur } \\
\text { l'eau gélosée après } 40 \mathrm{~h} \\
\text { d'incubation }\end{array}$ & $\begin{array}{l}\text { La majorité sont simples } \\
(50-80 \%)\end{array}$ & $\begin{array}{l}\text { La majorité sont ramifiés } \\
(50-80 \%)\end{array}$ & $\checkmark$ \\
\hline
\end{tabular}

1. La biométrie des macroconidies d'été, l'aspect cultural et la croissance mycélienne en fonction de la température correspondent pour les deux espèces. 


\section{RÉFÉRENCES}

Bresadola, J. 1893. Fungi aliquot saxonici novi lecti a cl. W. Krieger. Hedwigia 32 : 32-33.

Butin, H. 1983. Krankheiten der Wald-und Parkbäume. Georg Thieme Verlag, Stuttgart, p. 65-66.

Conners, J.L. 1967. An annotated index of plant diseases in Canada. Can. Dept. of Agriculture. Publ. 1251. Ottawa, p. 255.

Farr, D.F., G.F. Bills, G.P. Chamuris et A.Y. Rossman. 1989. Fungi on plants and plant products in the United States. The Am. Phytopathol. Soc. APS Press, Minnesota, p. 510 et 778 .

Hill, J.T., D.J. Ormrod et R.J. Copeman. 1977. Weeping willow blight in coastal British Columbia. Can. Plant Dis. Surv. 57 : 7174.

Lanier, L., P. Joly, P. Bondoux et A. Bellemère. 1976. Mycologie et pathologie forestières. Tome II. Masson, Paris, p. 380382.

Marks, G.C., B.A. Further et N.E.M. Walters. 1982. Tree diseases in Victoria. Forests Commission (ed.), Victoria, Australia. Sec. 18, p. 62.

Meirleire, H. 1978. Une grave maladie des saules pleureurs. Phytoma 299 : 22-23.

Nattrass, R.M. 1930. A note on two Marssonina diseases on willows. Government Press, Cairo. Bulletin No. 99 : 1-19.
Nival, M. 1972. Étude du Marssonina salicicola en microscopie électronique. Ann. Univ. ARERS, Reims (France), 10(2): 57-66.

Rimpau, R.H. 1961. Untersuchungen über die Gattung Drepanopeziza (Kelb.) v. Höhn., Phytopathol. Z. 43 : 257-306.

Siskina, A.K. et C.I. Mamykasvili. 1978. [L'Anthracnose des saules pleureurs (Salix babylonica L.) dans les parcs de Tbilisi] Mikol. Fitopatol. 12 : 151-153.

Tzavella-Klonari, K., M.D. Aggelaki et D.A. Karidimos. 1997. First report of anthracnose on weeping willow in Greece. Plant Dis. $81: 960$

Vegh, I. 1974. Contribution à l'étude biologique du Marssonina salicicola (Bres.) Magn. Ann. Phytopathol. 6 : 309-339.

Vegh, I. et M. Bourgeois. 1968. Sur trois champignons parasites des saules en France. Revue Horticole, Extrait No. 2., 285 : 1563-1568.

Vegh I. et J. Velastegui. 1983. Étude comparée de trois espèces de Marssonina salicicoles. Cryptogam. Mycol. 4 : 345-351.

Vujanovic, V. et Z. Vucinic. 1992. Marssonina salicicola, parasite des saules au Monténégro. Symposium Yougoslave sur la Protection des Plantes. Vrnjacka Banja, Yougoslavie. Résumé, Vol. 1, p. 335. 